\title{
Migración Haitiana en tránsito por Ecuador*
}

\author{
Andrés López Rivera ${ }^{* *}$ y Jana Wessel ${ }^{* * *}$
}

\section{Introducción}

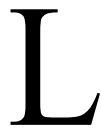

a migración haitiana es un fenómeno escasamente estudiado en el contexto sudamericano. Recientemente, a consecuencia del incremento de los movimientos migratorios a los que dio lugar el devastador terremoto de 2010 en Haití, se han realizado los primeros estudios sobre los flujos migrantes de este país en la región. En su mayoría, estos estudios se centran prioritariamente en Haití como país de origen y Brasil como país de destino desde un análisis de push factors y pull factors. Sin embargo, poca investigación se ha realizado en torno a los países de tránsito por los que circula el flujo migrante haitiano en Sudamérica, en particular Ecuador. La migración en tránsito en sí ha sido poco investigada debido a que se la considera como un fenómeno marginal y transitorio. Al mismo tiempo, el concepto de migración en tránsito es problemático y su aplicación conlleva cierta dificultad dada su ambigüedad y politización. ${ }^{1}$

* Ponencia presentada en el I Congreso Interinstitucional de Ciencias Políticas y Relaciones Internacionales (CIPRI), Quito-Ecuador. El documento se inscribe en el marco del proyecto de investigación "Hacia un nuevo enfoque de movilidad humana en el siglo XXI", de la carrera de Ciencias Políticas y Relaciones Internacionales, Universidad de Los Hemisferios.

** Investigador Doctoral, Max Planck Institute for the Study of Societies, Alemania. Master en Ciencia Política y Gobierno, London School of Economics and Political Science, Reino Unido. Profesor, Facultad de Ciencias Políticas y Jurídicas, Universidad de los Hemisferios, Ecuador; ‘alr@mpifg.de〉.

*** Máster en Comunicación para el Desarrollo, Malmö Universiteit, Suecia. Miembro del equipo de trabajo social y terapia, Caritas, Ecuador.

1. Michael Collyver y Hein de Hass, "Developing Dynamic Categorisations of Transit Migration". Population, Space and Place, No.18 (2012): 468-481; Franck Düvell, "Transit Migration: A Blurred and Politicised Concept”, Population, Space and Place 18, No. 4 (2012): 415-427. 


\section{Migración en tránsito}

La "migración en tránsito" es una categoría cada vez más presente en los discursos académicos, mediáticos y políticos. Si bien no existe una definición universalmente aceptada de migración en tránsito, es posible encontrar algunas definiciones en diversos documentos de organizaciones internacionales. Por ejemplo, la Comisión de las Naciones Unidas para Europa (CEPE) define la migración en tránsito como la "migración a un país con la intención de buscar la posibilidad de emigrar a otro país como país de destino final". ${ }^{2}$ De manera similar, la Organización Internacional para las Migraciones (OIM) provee una definición en su Estudio de la migración en tránsito en Azerbaiyán ${ }^{3}$ en el que se especifica que "Los migrantes en tránsito se definen como los extranjeros que permanecen en el país durante algún período de tiempo, mientras que deseen migrar en forma permanente a otro país". A las definiciones provenientes de organizaciones se suman definiciones formuladas por académicos, por ejemplo la definición de Ivakhniouk ${ }^{4}$ según la cual la migración en tránsito es "una estancia temporal, de corto plazo, de un migrante en su trayecto desde un país de origen a un país de destino".

Desde estas definiciones se esboza un concepto que en principio lograría replantear una concepción dicotómica de la migración y captar de mejor manera la complejidad del fenómeno migratorio. En efecto, al integrar el lapso de tránsito al análisis, se captaría una dimensión obliterada por la premisa según la cual la migración sería un proceso linear entre el país de origen y el país de destino, o un proceso que encierra dos facetas: la emigración y la inmigración. El "tránsito" se entiende como la estancia temporal o el trayecto entre dos países.

De las definiciones antes mencionadas cabe recalcar tres cuestiones que requieren mayor análisis. En primer lugar, algunas definiciones enfatizan el aspecto subjetivo del tránsito (p. ej. OIM 2003), es decir la voluntad del migrante de continuar el viaje, mientras que otras tratan éste como un hecho objetivo (p. ej. Ivakhniouk). En segundo lugar, las definiciones en su mayoría no

2. CEPE, Boletín de Migración Internacional, No. 3 (1993): 7. Traducción realizada por los autores.

3. IOM, The Next Stop is: Study on Transit Migration Through Azerbaijan, (Baku: IOM, 2003).

4. Irina Ivakhniouk, "Analysis of Economic, Social, demographic and Political basis of Transit Migration in Russia-Moscow Case" (ponencia, Regional Conference on 'Migration in Transit Countries: Sharing responsibility for management and protection, Estambul, 30 de septiembre-1 de octubre de 2004): 6 . 
especifican la duración máxima que englobaría la migración en tránsito. Por último, algunas definiciones entienden el "tránsito" como la estancia temporal en un país distinto a los países de origen y de destino, mientras que otros no precisan si el tránsito involucra un tercer país.

De forma preliminar, caben tres observaciones en relación con los tres puntos antes mencionados. Respecto al primero, consideramos que el "tránsito" no se puede analizar principalmente como un hecho subjetivo, ya que el aspecto volitivo, que se podría entender no solamente como una decisión sino incluso como una mera añoranza, es excesivamente ambiguo y omnipresente en la experiencia migratoria. En cuanto al segundo punto, tomando en cuenta los aspectos subjetivos y objetivos de la migración en tránsito, se evidencia una imposibilidad de definir una duración fija. En efecto, si bien la migración en tránsito se refiere a un fenómeno de corta duración, existen casos en los cuales se prolonga indefinidamente. Por último, respecto al tercer punto, consideramos que la migración en tránsito no es una categoría relevante a menos que involucre un tercer país distinto al país de origen y de destino. Si la migración en tránsito no incluyera un tercer país, entonces sería una categoría tautológica puesto que toda migración supone el tránsito de un lugar a otro. Ahora bien, esta última observación no faculta necesariamente la utilización de la categoría "país de tránsito" que, como señala Düvell, ${ }^{5}$ es ambigua y excesivamente politizada. En efecto, la categoría de país de tránsito se aplica arbitrariamente a países de la periferia que son atravesados por flujos migratorios.

El concepto de migración en tránsito es tanto más problemático cuanto que se lo asocia con la migración irregular o ilegal. ${ }^{6}$ Una ilustración explícita de esto es el documento de $\mathrm{CEPE}^{7}$ antes citado que identifica la migración en tránsito con "flujos de migrantes irregulares e ilegales desde el Tercer Mundo y de los países de Europa del Este”. La vinculación arbitraria entre migración en tránsito y migración irregular/ilegal es inherentemente problemática en la medida en que conlleva graves consecuencias para la política de control migratorio del tránsito. A partir del caso europeo, Düvell ${ }^{8}$ establece que el uso de la categoría de "migración en tránsito" se encuentra estrechamente ligada a la

\footnotetext{
5. Düvell, "Transit Migration: A Blurred and Politicised Concept".

6. Collyver y Hass, "Developing Dynamic Categorisations of Transit Migration".

7. CEPE, Boletín de Migración Internacional.

8. Düvell, "Transit Migration: A Blurred and Politicised Concept".
} 
externalización del control migratorio de la Unión Europea (UE). En este sentido, si bien la categoría de migración en tránsito provee cierta precisión descriptiva de la fase intermedia entre la emigración y la inmigración, es problemática dada su ambigüedad y politización.

\section{Migración haitiana}

Históricamente la migración haitiana se ha dado por factores estructurales de índole política y económica que han sumergido al país caribeño en crisis recurrentes: golpes de Estado, regímenes dictatoriales, ocupaciones extranjeras, aislamiento internacional y sanciones comerciales. A esto se han sumado desastres naturales que han sumergido al país en crisis humanitaria. Las consecuencias de estas crisis se han visto reflejadas en lo que se conoce como la diáspora haitiana, la cual se puede observar a través de la periodización histórica de la migración haitiana. En el siglo XX, Anglade9 identifica dos "olas migratorias": la primera de 1915 a 1935 y la segunda de 1965 a 1985. El primer flujo migratorio se dirige principalmente a Cuba y la República Dominicana, mientras que el segundo se dirige predominantemente a Estados Unidos y, en una menor medida, a Canadá, Francia, Las Bahamas, y distintos países de América Latina y África.

Siguiendo a Saint-Hubert, ${ }^{10}$ se pueden identificar cuatro fases principales desde el inicio de la "segunda ola" estudiada por Anglade. La primera fase (1965-1977) se dirige principalmente a los Estados Unidos, país que recibe alrededor de 7.500 migrantes procedentes de Haití. Esta fase se caracteriza por ser predominantemente una migración de clase media que se da en condiciones regulares. La segunda fase (1978-1985) se dirige igualmente en su mayoría a los Estados Unidos, pero se trata esta vez de una migración irregular/ ilegal de clases bajas que llegan a las costas estadounidenses por vía marítima en frágiles embarcaciones dando lugar así al fenómeno de boat people. La tercera (1987-1992) y la cuarta fase (desde 1993) se caracterizan por el asentamiento y la estabilización de la migración haitiana en los Estados Unidos.

9. Georges Anglade, "Les Haïtiens dans le Monde", 2005. 〈http://ile-en-ile.org/georges-anglade-les-haitiens-dans-le-monde/s.

10. Francis Saint-Hubert, "La Migration Haïtienne, un défi à relever", Migration Policy Practice 2, No. 2 (2012): 14-15 
- Figura 1. La migración haitiana en el mundo

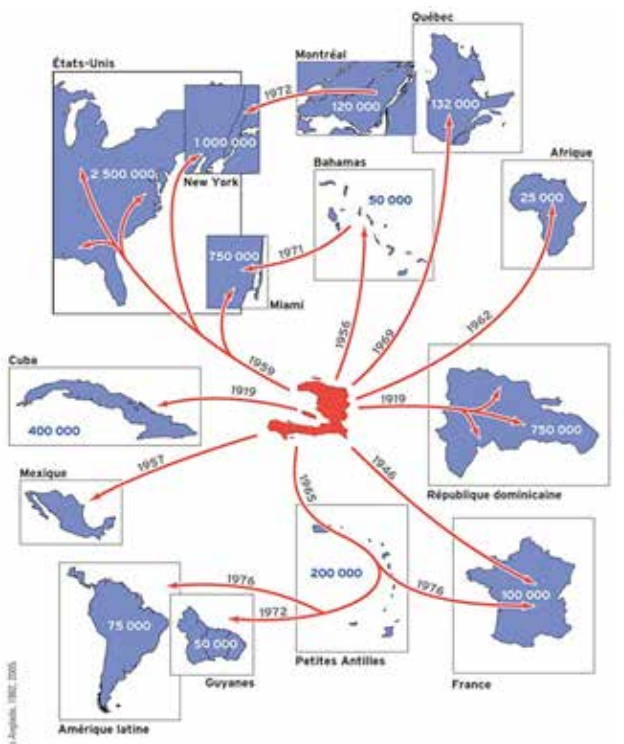

Fuente: Anglade, “Les Haïtiens dans le Monde” (2005).
A estas cuatro fases se podría incluir una quinta que inicia en 2010 y continúa en la actualidad.

En efecto, los factores estructurales que han provocado las distintas fases de emigración haitiana han sido agravados por factores coyunturales, en particular la crisis humanitaria generada por el terremoto de 2010. Entre los desastres naturales que afectaron a Haití, este ha sido el más devastador ya que los daños provocados han incapacitado al país frente a desastres naturales posteriores como, por ejemplo, el huracán Sandy de 2012. ${ }^{11}$ La crisis humanitaria tuvo por efecto el incremento de los flujos migra-

torios hacia destinos que no constan entre los más comunes de la diáspora haitiana. En este sentido, sería posible decir que la migración haitiana se encuentra en una quinta fase que inicia en 2010 y se caracteriza por la aparición de nuevos destinos, en particular Brasil.

\section{Brasil: país de destino}

La presencia de Brasil en Haití adquiere un rol significativo en especial desde 2004, año en el que asume el liderazgo de la Misión de las Naciones Unidas para la Estabilización en Haití (MINUSTAH). A esto se suma la presencia de organizaciones no-gubernamentales (ONG) y empresas brasileñas que realizan acciones humanitarias y proyectos de desarrollo y reconstrucción posterremoto. ${ }^{12}$ Brasil se forja así una imagen positiva entre la población

11. Gabriela Bernal Carrera, “¿Por qué migrar? Algunos apuntes sobre las viejas y nuevas heridas de Haiti”. En OIM, La Migración Haitiana hacia Brasil: Características, oportunidades y desafios, Cuadernos Migratorios, No. 6, (Buenos Aires: OIM, 2014): 33-50.

12. Isaias Albertin de Moraes, Carlos Alberto Alencar y Beatriz Rodriguez Bessa Mattos, "A migracao haitiana para o Brasil: causas e dasafios", Conjuntura Austral 4, No. 20 (2013): 95-114. 
haitiana. Adicionalmente, Brasil figura como un país de oportunidades especialmente a partir del momento en el que se convierte en anfitrión de la Copa FIFA 2014 y los Juegos Olímpicos 2016, eventos que requerían de mano de obra para la construcción de nuevas infraestructuras. Al mismo tiempo, en este periodo los controles fronterizos de los Estados Unidos incrementaron, lo cual fungió como un factor de disuasión para la migración de haitianos a este país. ${ }^{13}$ En contraposición, Brasil adoptó políticas de visado favorables a la migración haitiana, incluyendo la no deportación de inmigrantes irregulares. ${ }^{14}$ Estos factores contribuyeron a que, tras el terremoto de 2010, Brasil se convirtiera en uno de los principales países de destino de la migración haitiana.

Es importante notar que en el periodo previo al terremoto, la inmigración haitiana a Brasil era cuantitativamente insignificante. Posteriormente, esta inmigración creció en 2010 y se intensificó entre 2011 y 2012. Se estima que en este periodo ingresaron alrededor de 4.000 haitianos indocumentados a Brasil principalmente por los estados de Acre y Amazonas, pero también por Roraima, Mato Grosso y Amapá. ${ }^{15}$ En este periodo, los inmigrantes haitianos que llegaron a Brasil buscaron acogerse a la figura de refugio. La inaplicabilidad del refugio en el caso de los haitianos hizo que el gobierno decidiera adoptar la Resolución Recomendada No. 08/06 en abril de 2011, la cual permitió a los ciudadanos haitianos obtener un permiso de residencia en Brasil por razones humanitarias. Posteriormente, en enero de 2012, se adopta la Resolución No. 97 la cual prevé que el gobierno brasileño otorgue 1.200 visas anuales a ciudadanos haitianos a través de su consulado en Puerto Príncipe (Haití). Además, se realiza una entrega masiva de visas a ciudadanos haitianos que se encontraban en el territorio brasileño anteriormente. Estas medidas se amplían mediante la Resolución Normativa 102/2013 que elimina la restricción de 1.200 visas anuales y ya no restringe la concesión de visas al Consulado de Brasil en Puerto Príncipe. ${ }^{16}$

13. Gabriela Bernal Carrera, "La migración haitiana hacia Brasil: Ecuador, país de tránsito". En OIM, La Migración Haitiana hacia Brasil: Características, oportunidades y desafios, Cuadernos Migratorios, No. 6, (Buenos Aires: OIM, 2014): 67-82.

14. Tobías Metzner, "La migración haitiana hacia Brasil: estudio en el país de origen”. En OIM, La Migración Haitiana hacia Brasil: Características, oportunidades y desafios, Cuadernos Migratorios, No. 6, (Buenos Aires: OIM, 2014): 15-30.

15. Albertin de Morales, Alencar y Rodriguez Mattos, "A migracao haitiana para o Brasil: causas e dasafios".

16. Metzner, "La migración haitiana hacia Brasil: estudio en el país de origen"; Albertin de Moraes, Alencar y Rodriguez Mattos, "A migracao haitiana para o Brasil: causas e dasafios". 
De acuerdo al Ministerio de Asuntos Exteriores, Brasil emitió hasta julio de 2015 aproximadamente 26.000 visas humanitarias a los inmigrantes haitianos. De estas, 20.000 se emitieron en Puerto Príncipe (Haití) y otras 6.000 en Quito (Ecuador). ${ }^{17}$ Según estas cifras, la migración haitiana por medios regulares que atravesó Ecuador en el periodo comprendido entre 2010 y 2015 se dirigió a Brasil. Sin embargo, a esto se suman flujos migratorios irregulares que tenían igualmente como destino Brasil y que se dirigían primeramente desde Ecuador a Perú por vía terrestre para finalmente ingresar a Brasil. Se desconoce qué porcentaje del total de migrantes haitianos en tránsito hacia Brasil representa la cifra antes citada, debido a que la migración irregular no se puede documentar. Por último, a estas cifras se suma igualmente un número desconocido de migrantes haitianos en tránsito cuyos países de destino fueron otros, especialmente Chile y Argentina. ${ }^{18}$

\section{Ecuador: país de tránsito}

Históricamente la inmigración haitiana a Ecuador ha sido marginal. Solamente desde 2008 la entrada de haitianos al país incrementó ostensiblemente. Desde entonces, Ecuador se convirtió progresivamente en un punto de paso en el trayecto de los haitianos hacia Brasil. En efecto, la ruta migratoria de los haitianos parte desde Puerto Príncipe (Haití) o Santo Domingo (República Dominicana), en dirección a Ecuador por vía aérea. Posteriormente, los migrantes haitianos continúan su viaje por vía terrestre o aérea hacia Perú para finalmente atravesar la frontera de Brasil. En ciertos casos, los haitianos que obtenían visado en el consulado de Brasil de Quito, se dirigían directamente a Brasil omitiendo así el paso por territorio peruano. ${ }^{19}$ La estancia en Ecuador es variable puesto que en ciertos casos se limita a pocos días, mientras que en otros se extiende a varios meses. ${ }^{20}$ Una gran parte de haitianos que pasan por Ecuador tramitaban su visa en la embajada de Brasil donde se enfrentaban

17. Governo do Brasil, "Concessao de visto humanitario para haitianos é prorrogada", 2015. 〈http://www. brasil.gov.br/cidadania-e-justica/2015/08/concessao-de-visto-humanitario-para-haitianos-e-prorrogada).

18. Iréri Ceja Cárdenas, "Migraciones haitianas en la región andina", Boletín Andina Migrante, No. 19 (2015): 2-13.

19. Ibíd.

20. Tania Vázquez, Erika Busse y Lorena Izaguirre, "La migración haitiana en Perú y su tránsito hacia Brasil”. En OIM, La Migración Haitiana hacia Brasil: Características, oportunidades y desafios, Cuadernos Migratorios, No. 6, (Buenos Aires: OIM, 2014): 83-105. 
- Figura 2. Ruta migratoria de haitianos hacia Brasil

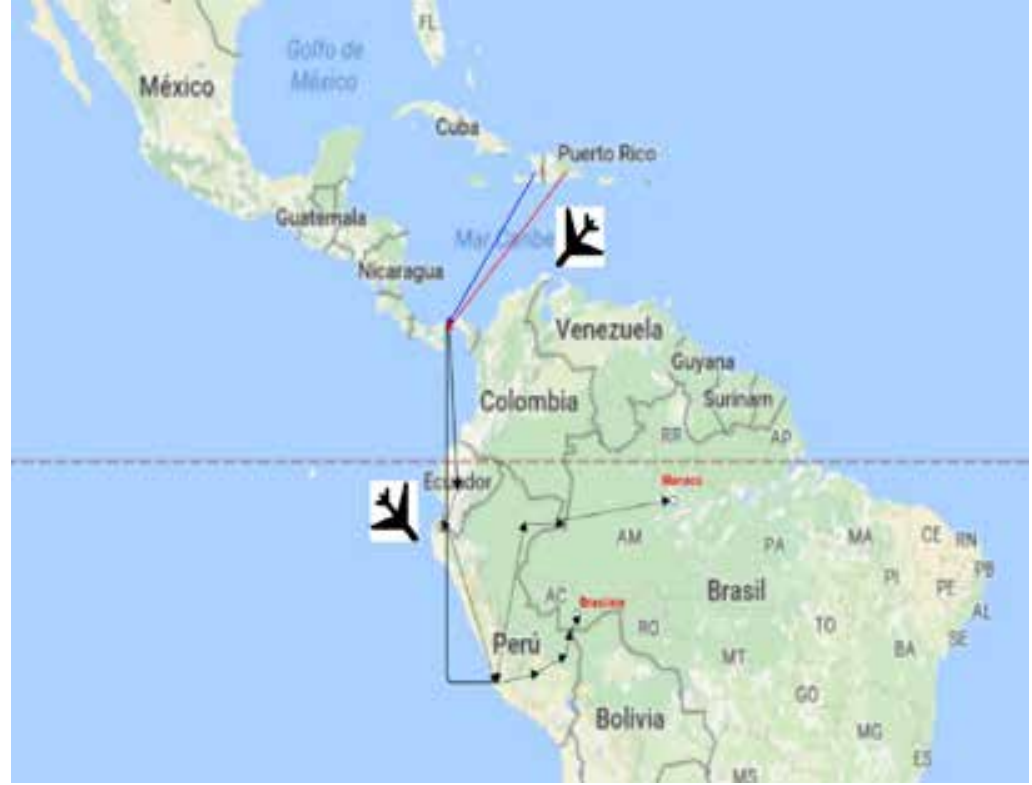

Fuente: Duval Fernandes, Rosita Milesi y Andressa Farias. Do Haiti para o Brasil: o novo uxo migratório, Refúgio, Migrações e Cidadania, Caderno de Debates, No. 6 (20II).

Elaboración: autor.

con problemas debido al hecho de que las citas otorgadas excedían el plazo de la visa en ciertos casos teniendo como resultado la venta ilícita de turnos. ${ }^{21}$

En base a los datos del Instituto Nacional de Estadísticas y Censos (INEC) (ver tabla 1), es posible identificar dos repuntes en la entrada de migrantes haitianos al país. El primer repunte se da entre 2008 y 2009, periodo en que el número de entradas pasa de 270 a 1.257. Este primer aumento se explica por la reforma a la política migratoria ecuatoriana, en particular la eliminación de visado de turismo para ciudadanos extranjeros en junio de 2008. La eliminación del visado se inscribe en la adopción de un nuevo paradigma migratorio establecido por la Constitución de 2008, en el cual se adoptan los principios de ciudadanía universal, libre movilidad, eliminación de ilegalidad de la condición migratoria beneficiando a las personas en movilidad humana (Artículo 416). El segundo repunte se da entre 2012 y 2013. En este periodo el número

21. Ceja Cárdenas, "Migraciones haitianas en la región andina". 
. Tabla 1. Entradas y salidas de haitianos a Ecuador entre 1998-2015.

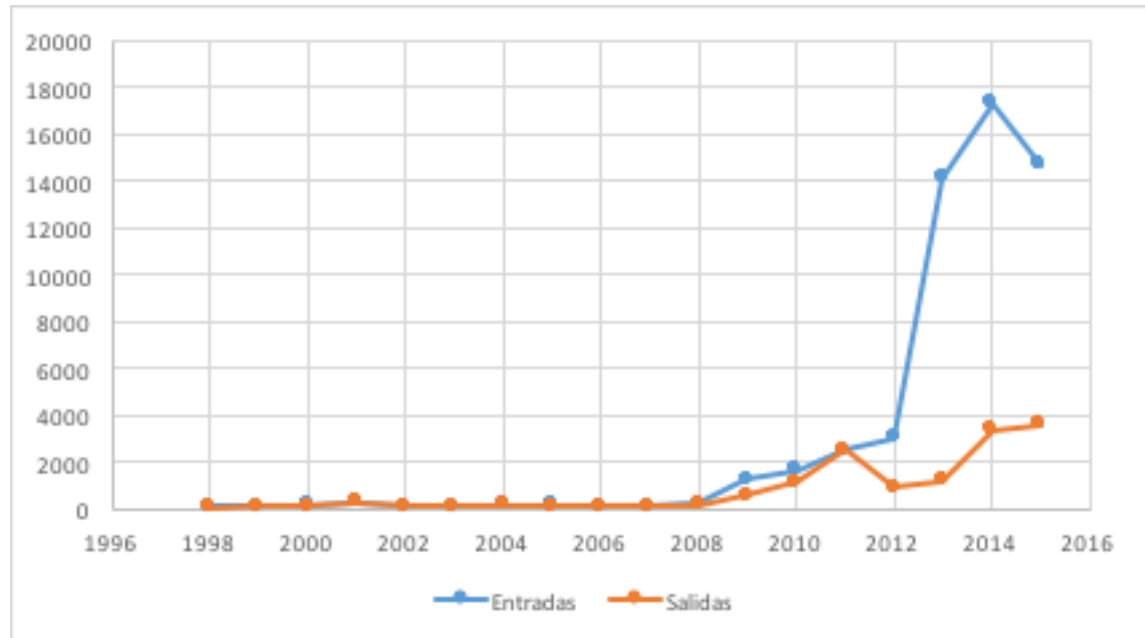

Fuente: Instituto Nacional de Estadísticas y Censos (INEC).

Elaboración: autor.

de entradas pasa de 3.023 a 14.099. Las razones de este segundo aumento son varias. Por un lado, Brasil inicia a otorgar visas humanitarias desde el consulado de Quito en 2013. Por otro lado, en enero de 2012 Perú implementa una visa temporal de turista para ciudadanos haitianos de tal manera que estos se ven obligados a dirigirse primeramente a Ecuador. A esto se suma el paso del huracán Sandy de 2012 por Haití lo cual agudizó la grave crisis humanitaria provocada por el terremoto de 2010.

El número de entradas de haitianos a Ecuador ha ido incrementando con relación al número de salidas. Es decir, los datos parecerían indicar que Ecuador está pasando de ser un "país de tránsito" a ser un "país de destino" para los migrantes haitianos tomando en cuenta que se observan menos salidas que entradas de haitianos a partir de 2011. No obstante, es importante considerar que el número oficial de salidas no incluye a las salidas clandestinas del país y que existen inmigrantes haitianos que han prolongado su estancia en Ecuador dada la demora en los trámites de visado. A fin de determinar las causas del desfase entre número de entradas y número de salidas desde 2011, es necesario recabar más información sobre el tipo de visa -o situación irregular- bajo el cual los ciudadanos haitianos permanecen en el territorio ecuatoriano. Así mismo se requiere observar el desarrollo de esta tendencia en los próximos años. 
Durante el periodo considerado, se documentaron diversos impedimentos a la inmigración haitiana en Ecuador. Primero, en marzo de 2013 se propuso la exigencia de presentar una carta de invitación para ciudadanos haitianos que deseen ingresar al país; sin embargo la medida se suspendió antes de su implementación. ${ }^{22}$ Segundo, en agosto de 2015 se implementó la obligación de un "Formulario Único de Validación Turística" para los ciudadanos haitianos que deseen ingresar al territorio ecuatoriano. ${ }^{23} \mathrm{El}$ formulario recaba información sobre previas estancias en Ecuador y sobre las actividades turísticas a realizarse. Por último, se ha reportado que los agentes de migración del aeropuerto de Quito, solicitaban discrecionalmente demostrar solvencia económica a los ciudadanos haitianos para poder ingresar en calidad de turistas. ${ }^{24}$

\section{¿Nuevos flujos migratorios?}

La crisis económica en Brasil que inició en 2015 y se agudizó en 2016 ha sido uno de los factores centrales de la salida de migrantes haitianos con destino a otros países en especial Estados Unidos. Como explica Salcedo, ${ }^{25}$ aún no es posible determinar el impacto real de la crisis en los flujos migrantes haitianos. Sin embargo, a fines de 2016, se dan los primeros indicadores de que un nuevo flujo migratorio está emergiendo. La ruta desde Brasil hacia los Estados Unidos atraviesa los siguientes países: Perú, Ecuador, Colombia, Panamá, Costa Rica, Nicaragua, Honduras, Guatemala y México. Según informes de prensa, en Estados Unidos el mayor incremento en la llegada de haitianos indocumentados se ha registrado en la ciudad fronteriza de San Diego, California. La llegada de haitianos a esta ciudad pasó de 339 en 2015 a 4.346 en $2016 .{ }^{26}$

En esta ruta emergente de la migración haitiana Ecuador figura nuevamente como un país de tránsito. Al tratarse de un flujo irregular, los migrantes se

22. "Gobierno de Ecuador suspende carta de invitación para haitianos", La Hora (Quito), 30 de Marzo de 2013.

23. Ecuador Ministerio de Relaciones Exteriores y Movilidad Humana, "Aplicación del Formulario Único de Validación Turística para ciudadanos haitianos", 2015. 〈http://www.cancilleria.gob.ec/aplicacion-del-formulario-unico-de-validacion-turistica-para-ciudadanos-haitianos/>.

24. Ceja Cárdenas, "Migraciones haitianas en la región andina".

25. Adriana Salcedo, "Tendencias de las migraciones en el Caribe Insular". En Allison Petrozziello, edit., Estado de las migraciones que atañen a la República Dominicana 2015. OBMICA (Santo Domingo: Editora Búho, 2016).

26. "New Migrations: Haitians carve a dangerous 7000 mile path to the US". Miami Herald (Miami), 24 de septiembre de 2016. 
- Figura 2. Ruta migratoria de haitianos desde Brasil hacia Estados Unidos

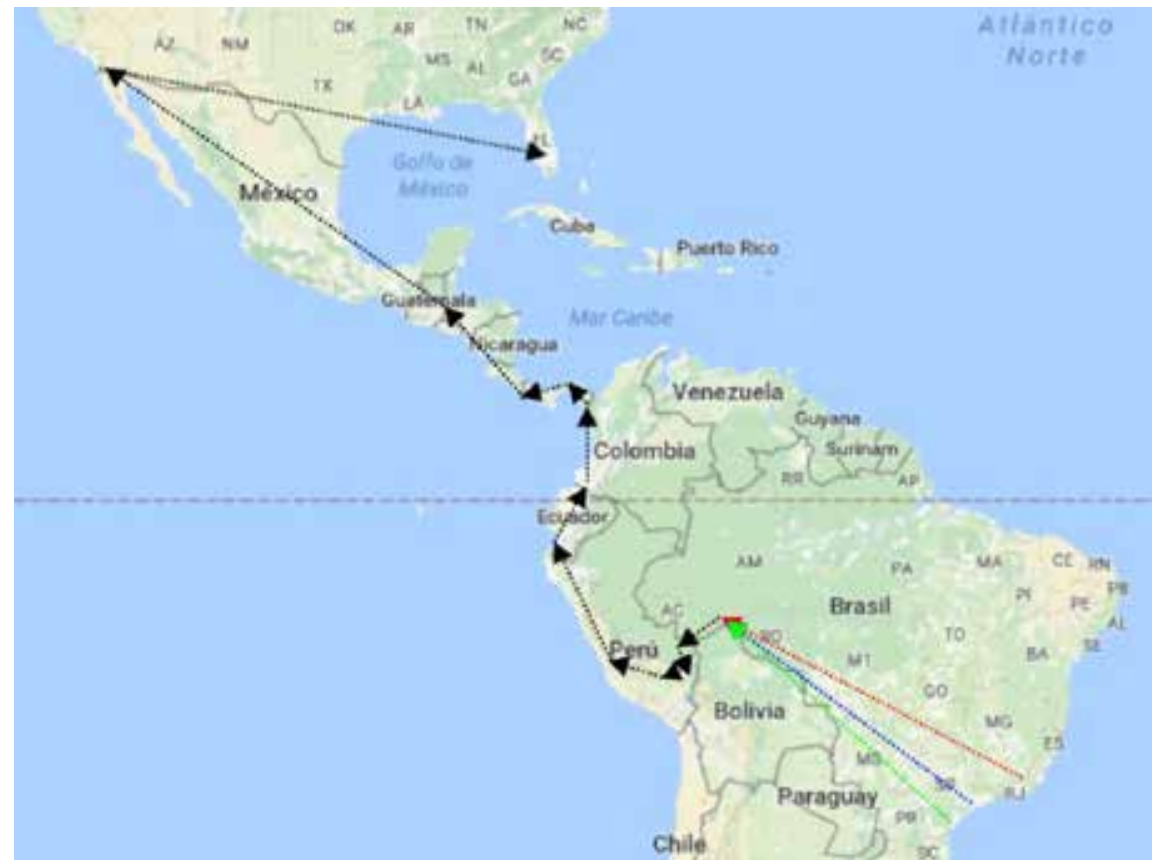

Fuente:"New Migrations: Haitians carve a dangerous 7000 mile path to the US”. Miami Herald (Miami), 24 de septiembre de 2016.

Elaboración: autor.

encuentran en situación de mayor vulnerabilidad y en varias ocasiones se han registrado deportaciones desde Ecuador y otros países que se encuentran en la ruta de paso de los migrantes. En agosto de 2016, por ejemplo, se informó de un grupo de aproximadamente 150 haitianos que se encontraban "varados" en Rumichaca, zona fronteriza entre Ecuador y Colombia, y que fueron posteriormente deportados. ${ }^{27}$ Aunque la información sobre la conformación de este nuevo flujo migratorio es escasa, preliminarmente es posible evidenciar se la migración haitiana en la subregión sudamericana se ha precarizado principalmente a causa de la crisis económica en Brasil.

27. "Haitianos varados en frontera serán deportados en 48 horas”. El Comercio (Quito), 6 de agosto 2016. 


\section{Consideraciones finales}

La migración haitiana en tránsito por Ecuador es un fenómeno de dinámicas complejas en constante cambio. Al inicio del presente análisis se señalaron tres aspectos problemáticos en las definiciones del concepto de migración en tránsito. En base a lo expuesto a través del caso haitiano es posible identificar una serie de interrogantes para futuras investigaciones. En primer lugar, en lo que se refiere a los aspectos objetivos y subjetivos del tránsito, desde un análisis previo de la migración haitiana en tránsito surge principalmente una interrogante: si el tránsito se define como la expectativa de movilidad del migrante, ¿cómo se entiende la permanencia de facto y viceversa? Se evidencia entonces la necesidad de investigar la experiencia migratoria contrastando las aspiraciones subjetivas de la persona en situación de movilidad con las restricciones objetivas a las que ésta se enfrenta.

En segundo lugar, un punto relacionado con el anterior es la duración del tránsito. Desde la experiencia haitiana se evidencia que en ciertos casos el tránsito se prolonga indefinidamente. De ahí la necesidad de contrastar la expectativa individual de movilidad frente a las limitaciones impuestas por la situación del migrante. La duración del "tránsito" trae consigo implicaciones sustanciales para la situación social, económica y legal del migrante. En efecto, un tránsito prolongado que excede la duración de la estadía permitida es la causa de la situación de irregularidad del sujeto migrante. Es decir, la asociación del concepto de tránsito con la migración ilegal no es solamente arbitraria como se señaló en un principio, sino que es falsa puesto que en ciertos casos se da una situación inversa en la que la ilegalización del migrante es causada por la prolongación indefinida del tránsito.

En cuanto al último punto, es necesario problematizar la asociación de la categoría de país de tránsito. En el caso haitiano esto se evidencia en dos aspectos. Por un lado, el país de tránsito puede convertirse en país de destino. Por otro lado, al igual que en el caso europeo, el caso latinoamericano nos enseña que la categoría de país de tránsito está inscrita en una lógica de externalización de las fronteras del país de destino. En este sentido, el "país de tránsito" aparece como el país subdesarrollado de la periferia que está obligado a aumentar el control migratorio para evitar que los flujos de migrantes lleguen a las fronteras del país desarrollado al que se dirigen los mismos. Las recientes deportaciones de haitianos en el trayecto hacia los Estados Unidos son un 
indicador de este fenómeno. En suma, es necesario replantear el rol de Ecuador como país de tránsito reconsiderando las dinámicas complejas de la migración en tránsito.

\section{Bibliografía}

Albertin de Moraes, Isaias, Carlos Alberto Alencar de Andrade y Beatriz Bessa Mattos. "A migração haitiana para o Brasil: causas e dasafios”. Conjuntura Austral, 4 (2013): 95-114.

Anglade, Georges. "Les Haïtiens dans le Monde", 2005. 〈http://ile-en-ile.org/georges-anglade-les-haitiens-dans-le-monde/s.

Bernal Carrera, Gabriela. "La migración haitiana hacia Brasil: Ecuador, país de tránsito". En OIM, La Migración Haitiana hacia Brasil: Características, oportunidades y desafios, Cuadernos Migratorios, No. 6. Buenos Aires: OIM, 2014. 67-82.

—. "¿Por qué migrar? Algunos apuntes sobre las viejas y nuevas heridas de Hait'”. En OIM, La Migración Haitiana hacia Brasil: Características, oportunidades y desafios, Cuadernos Migratorios, No. 6. Buenos Aires: OIM, 2014. 33-50.

Ceja Cárdenas, Iréri. "Migraciones haitianas en la región andina". Boletín Andina Migrante, No. 19 (2015): 2-13.

CEPE. Boletín de Migración Internacional, No. 3 (1993).

Collyver, Michael y Hein de Hass. "Developing Dynamic Categorisations of Transit Migration”. Population, Space and Place 18, No. 4 (2010): 468-481.

Düvell, Franck. "Transit Migration: A Blurred and Politicised Concept". Population, Space and Place 18, No. 4 (2012): 415-427.

Ecuador Ministerio de Relaciones Exteriores y Movilidad Humana. "Aplicación del Formulario Único de Validación Turística para ciudadanos haitianos", 2015. «http:// www.cancilleria.gob.ec/aplicacion-del-formulario-unico-de-validacion-turistica-para-ciudadanos-haitianos/>.

Fernandes, Duval, Rosita Milesi y Andressa Farias. "Do Haiti para o Brasil: o novo uxo migratório”. Cadernos de Debates Refúgio, Migrações e Cidadania 6, №. 6 (2011): 73-98.

"Gobierno de Ecuador suspende carta de invitación para haitianos". La Hora (Quito), 30 de Marzo de 2013.

Governo do Brasil, "Concessão de visto humanitario para haitianos é prorrogada", 2015. 〈http://www.brasil.gov.br/cidadania-e-justica/2015/08/concessao-de-visto-humanitario-para-haitianos-e-prorrogada.

"Haitianos varados en frontera serán deportados en 48 horas". El Comercio (Quito), 6 de agosto 2016.

IOM. The Next Stop is: Study on Transit Migration Through Azerbaijan. Baku: IOM, 2003. Ivakhniouk, Irina. "Analysis of Economic, Social, demographic and Political basis of Transit Migration in Russia-Moscow Case" (ponencia, Regional Conference on 'Migra- 
tion in Transit Countries: Sharing responsibility for management and protection, Estambul, 30 de septiembre-1 de octubre 2004).

Metzner, Tobías. "La migración haitiana hacia Brasil: estudio en el país de origen". En OIM, La Migración Haitiana hacia Brasil: Características, oportunidades y desafios, Cuadernos Migratorios, No. 6. Buenos Aires: OIM, 2014. 15-30.

"New Migrations: Haitians carve a dangerous 7000 mile path to the US". Miami Herald (Miami), 24 de septiembre de 2016.

Saint-Hubert, Francis. "La Migration Haïtienne, un défi à relever", Migration Policy Practice 2 No. 2 (2012): 14-15.

Salcedo, Adriana. "Tendencias de las migraciones en el Caribe Insular". En Allison Petrozziello, edit., Estado de las migraciones que atañen a la República Dominicana 2015. OBMICA. Santo Domingo: Editora Búho, 2016.

Vázquez, Tania, Erika Busse y Lorena Izaguirre. "La migración haitiana en Perú y su tránsito hacia Brasil". En OIM, La Migración Haitiana hacia Brasil: Características, oportunidades y desafios, Cuadernos Migratorios, No. 6. Buenos Aires: OIM, 2014: 83-105. 